University of Nebraska - Lincoln

DigitalCommons@University of Nebraska - Lincoln

CSE Technical reports

Computer Science and Engineering, Department of

2009

\title{
An Efficient Threshold-Based Power Management Mechanism for Heterogeneous Soft Real-Time Clusters
}

Leping Wang

University of Nebraska-Lincoln, lwang@cse.unl.edu

Ying Lu

University of Nebraska-Lincoln, ying@unl.edu

Follow this and additional works at: http://digitalcommons.unl.edu/csetechreports

Wang, Leping and Lu, Ying, "An Efficient Threshold-Based Power Management Mechanism for Heterogeneous Soft Real-Time Clusters" (2009). CSE Technical reports. 133.

http://digitalcommons.unl.edu/csetechreports/133

This Article is brought to you for free and open access by the Computer Science and Engineering, Department of at DigitalCommons@University of Nebraska - Lincoln. It has been accepted for inclusion in CSE Technical reports by an authorized administrator of DigitalCommons@University of Nebraska - Lincoln. 


\title{
An Efficient Threshold-Based Power Management Mechanism for Heterogeneous Soft Real-Time Clusters
}

\author{
Leping Wang, Ying Lu \\ Department of Computer Science and Engineering \\ University of Nebraska - Lincoln \\ $\{l w a n g, y l u\} @ c s e . u n l . e d u$
}

\begin{abstract}
With growing cost of electricity, the power management of server clusters has become an important problem. However, most previous researchers only address the challenge in homogeneous environments. Considering the increasing popularity of heterogeneous systems, this paper proposes an efficient algorithm for power management of heterogeneous soft real-time clusters. It is built on simple but effective mathematical models. When deployed to a new platform, the software incurs low configuration cost because no extensive performance measurements and profiling are required. To strive for efficiency, a threshold-based approach is adopted. In this paper, we systematically study this approach and its design decisions.
\end{abstract}

\section{Introduction}

Clusters of commodity-class PCs are widely used. When designing such a system, traditionally researchers have focused on maximizing performance. Recently, with a better understanding of the overall cost of computing [1], researchers have started to pay more attention to optimizing performance per unit of cost. According to [1], the total cost of ownership (TCO) includes the cost of cluster hardware, software, operations and power. As a result of recent advances in chip manufacturing technology, the performance per hardware dollar keeps going up. However, the performance per watt has remained roughly flat over time. If this trend continues, the power-related costs will soon exceed the hardware cost and become a significant fraction of the total cost of ownership.

To reduce power and hence improve the performance per watt, cluster power management mechanisms [6, $12,14,17,23,26]$ have been proposed. Most of them, however, are only applicable to homogenous systems. It remains a difficult problem to manage power for heterogeneous clusters. Two new challenges have to be addressed. First, according to load and server characteristics, a power management mechanism must decide not only how many but also which cluster servers should be turned on; second, unlike a homogenous cluster, where it is optimal to evenly distribute load among active servers, identifying the optimal load distribution for a heterogeneous cluster is a non-trivial task.

Department of Computer Science \& Engineering, University of Nebraska-Lincoln, Technical Report, TR-UNL-CSE-2009-0014 
A few researchers $[12,17]$ have investigated mechanisms to address the aforementioned challenges. However, their mechanisms require either an extensive performance measurement ("at most few hours for each machine" [17]) or a time-consuming optimization procedure [12]. These high customization costs are prohibitive, especially if these procedures need to be executed repetitively. Composed of a large number of machines, a cluster is very dynamic, where servers can fail, be removed from or added to it frequently. To achieve high availability in such an environment, a mechanism that is easy to be modified upon changes is essential. This paper proposes an efficient algorithm for power management (PM) of heterogeneous soft real-time clusters. We make two contributions. First, the algorithm is based on simple but effective mathematical models, which reduces customization costs of PM components to new platforms. Second, the developed online mechanisms are threshold-based. According to an offline analysis, thresholds are generated that divide the workload into several ranges. For each range, the power management decisions are made offline. Dynamically, the PM component just measures and predicts the cluster workload, decides its range, and follows the corresponding decisions. In this paper, we systematically investigate this low-cost efficient power management approach. Simulation results show that our algorithm incurs low overhead and leads to optimal power consumption.

The remainder of this paper is organized as follows. The related work is illustrated in Section 2. Sections 3 and 4 respectively present the models and state the problem. We discuss the algorithms in Section 5 and evaluate their performance in Section 6. Section 7 concludes the paper.

\section{Related Work}

Power management of server clusters $[2,6,15,16,23,26]$ has become an important problem. The authors of $[5,3]$ were the first to point out that cluster-based servers could benefit significantly from dynamic voltage scaling (DVS). Besides server DVS, dynamic resource provisioning (server power on/off) mechanisms were investigated in $[9,14]$ to conserve power in clusters.

The aforementioned research has all focused on homogeneous systems. However, clusters are almost invariably heterogeneous in term of their performance, capacity and power consumption [12]. Survey [4] discusses the recent work on power management for server systems. It lists power management of heterogeneous clusters as one of the major challenges.

Heath et. al. [12] considered proper cluster configuration and request distribution to optimize power and throughput in heterogeneous server clusters. Their mechanism takes characteristics of different nodes and request types into account. However, this approach depends on a time-consuming optimization procedure. Besides, it is not designed for real-time clusters.

Some researchers $[17,23,25]$ have investigated the power management problem for heterogeneous realtime systems. Among them, the most closely related work is by Rusu et. al. [17], where they investigated energy efficient real-time heterogeneous clusters. Like Heath et. al. [12], the authors of that paper [17] note 
that in heterogeneous clusters it may not be optimal in terms of power consumption to turn on just the smallest number of machines to satisfy the current load. They assume that servers in a heterogeneous cluster can be easily ordered with respect to their power efficiencies and thus servers are powered on/off following the power efficiency order to optimize power and provide real-time guarantees. However, our work shows that the proper order for switching on/off servers is not always obvious. In this paper, therefore, we systematically study different server ordering schemes (see Section 5.2 for details).

The approach by Rusu et. al. [17] also requires an extensive performance measurement, which needs to be carried out upon new installations, cluster upgrades or changes. Extensive performance measurements [17] and long optimization procedures [12] lead to high customization costs. To avoid these prohibitive costs, we propose in this paper a simple power management algorithm for heterogeneous soft real-time clusters. The algorithm is based on mathematical models that require minimum performance profiling. Instead of solving the optimization problem for every possible load, our algorithm derives thresholds, divides load into several ranges and determines the best cluster configuration formula for each workload range, leading to a time-efficient optimization procedure. Furthermore, our algorithm incurs low overhead and achieves optimal power consumption. We have published our preliminary work in a conference paper [21]. This journal paper significantly extends that work [21] — we tailor our power management algorithm and make it also applicable to clusters, where servers have significant switch on/off overheads and their CPUs only support discrete frequencies.

\section{Models}

In this section we present our models and state assumptions related to these models.

\subsection{System Model}

A cluster consists of a front-end server, connected to $\mathrm{N}$ back-end servers. We assume a typical cluster environment in which the front-end server does not participate in the request processing. The main role of the front-end server is to accept requests and distribute them to back-end servers. In addition, we deploy the power-management mechanism on the front-end server to enforce a server power on/off policy. Figure 1 shows a web server cluster example that fits our system model.

In a heterogeneous cluster, different back-end servers could have different computational capacities and power efficiencies. In the following, we describe their models. We assume processors on the back-end servers support dynamic voltage scaling and their operating frequencies could be continuously adjusted in the range $\left(0, f_{i \_m a x}\right]$. If a processor only supports discrete frequencies, we follow an approach similar to that proposed in [20] to approximate the desired continuous frequency setting by switching between two adjacent supported discrete frequency values. The capacity model relates the CPU operating frequency to the server's throughput and the power model describes the relation between the CPU frequency and the power consumption. While 
our approach could be generalized to different capacity and power models, in this paper we assume and use the following specific models to illustrate our method.

\subsection{Capacity Model}

We assume that the cluster provides CPU-bounded services. This is normal for most web servers because much of the data are already in memory $[5,17,24]$. Therefore, to measure the capacity of a back-end server its CPU throughput is used as the metric, which is assumed to be proportional to the CPU operating frequency. That is, the $i^{\text {th }}$ server's throughput, denoted as $\mu_{i}$, is expressed as $\mu_{i}=\alpha_{i} f_{i}$, where $\alpha_{i}$ is the CPU performance coefficient. Different servers may have different values for $\alpha_{i}$. With the same CPU frequency setting, the higher the $\alpha_{i}$ the more powerful the server is.

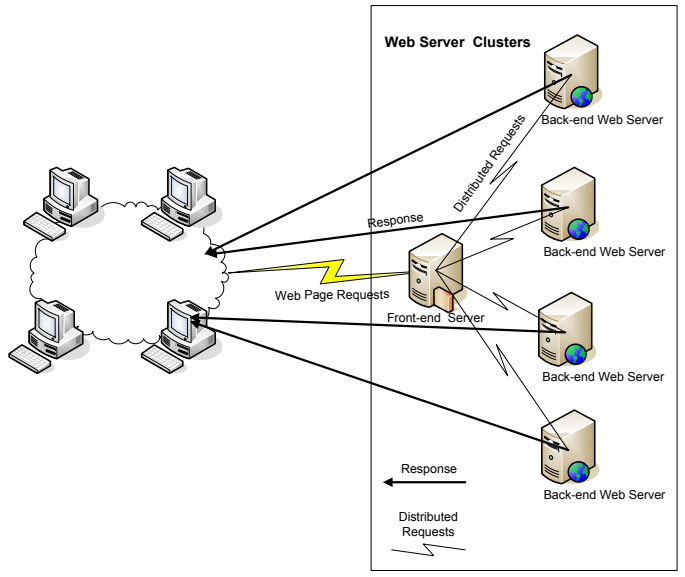

Figure 1. System Model

\subsection{Power Model}

The power consumption $P_{i}$ of a server consists of a constant part and a variable part. Similar to previous work $[9,6,13]$, we approximate $P_{i}$ by the following function:

$$
P_{i}=x_{i}\left(c_{i}+\beta_{i} f_{i}^{p}\right)
$$

where $x_{i}=1 / 0$ denotes the server's on/off state. When a server is off, it consumes no power; when it is on, it consumes $c_{i}+\beta_{i} f_{i}^{p}$ amount of power. In this model, $c_{i}$ denotes the constant power consumption of the server. It is assumed to include the base power consumption of the CPU and the power consumption of all other components. In addition, the CPU also consumes a power $\beta_{i} f_{i}^{p}$ that is varied with the CPU operating frequency $f_{i}$. In the remaining of this paper, we use $p=3$ to illustrate our approach.

Hence, in the cluster the power consumption of all back-end servers can be expressed as follows:

$$
J=\sum_{i=1}^{N} x_{i}\left[c_{i}+\beta_{i} f_{i}^{3}\right]
$$

Here, for the purpose of differentiation, $J$ is used to denote the cluster's power consumption while $P$ denotes a server's power consumption.

Following the aforementioned models, each server is specified with four parameters: $f_{i_{-} m a x}, \alpha_{i}, c_{i}$, and $\beta_{i}$. To obtain these parameters, only a little performance profiling is required. 


\section{Power Management Problem}

Given a cluster of $N$ heterogeneous back-end servers, each specified with parameters $f_{i-\max }, \alpha_{i}, c_{i}$, and $\beta_{i}$, the objective is to minimize the power consumed by the cluster while satisfying the following QoS requirement: $R_{i} \approx \hat{R}$, where $R_{i}$ stands for the average response time of requests processed by the $i^{t h}$ back-end server and $\hat{R}$ stands for the desired response time. The average response time $R_{i}$ is determined by the back-end server's capacity and workload. We use $\mu_{i}=\alpha_{i} f_{i}$ to denote the server's capacity and $\lambda_{i}$, the server's average request rate, to represent the workload. Thus, $R_{i}$ is a function of these two parameters, i.e., $R_{i}=g\left(\mu_{i}, \lambda_{i}\right)$. To enforce $R_{i} \approx \hat{R}$, we must control $\mu_{i}=\alpha_{i} f_{i}$ and $\lambda_{i}$ properly. As a result, the power management problem is formed as follows:

minimize

$$
J=\sum_{i=1}^{N} x_{i}\left[c_{i}+\beta_{i} f_{i}^{3}\right]
$$

subject to:

$$
\begin{cases}\sum_{i=1}^{N} x_{i} \lambda_{i}=\lambda_{\text {cluster }} & \\ x_{i}\left(1-x_{i}\right)=0, & i=1,2, \cdots, N \\ g\left(\alpha_{i} f_{i}, \lambda_{i}\right) \approx \hat{R}, & i=1,2, \cdots, N\end{cases}
$$

where $\lambda_{\text {cluster }}$ is the current average request rate of the cluster. We assume the cluster is not overloaded, that is, the average response time requirement $\forall i, g\left(\alpha_{i} f_{i}, \lambda_{i}\right) \approx \hat{R}$ is feasible for the cluster with a $\lambda_{\text {cluster }}$ request rate. ${ }^{1}$ The first optimization constraint guarantees that each request is processed by an active back-end server while the second constraint says a server is either in an on or an off state.

For the power management, the front-end component decides the server's on/off state $\left(x_{i}\right)$ and the workload distribution among the active servers $\left(\lambda_{i}\right)$. On the back-end, an approach that combines feedback control with queuing theoretic prediction, similar to that proposed in [18], is adopted to decide the CPU frequency setting to ensure the response time requirement. Accordingly, each active server adjusts its CPU operating frequency $f_{i}$ in the range $\left(0, f_{i_{-} \text {max }}\right]$. In the case where $\mathrm{CPU}$ frequency can only be set to some discrete values, to approximate the desired continuous frequency setting, we switch the CPU frequency at appropriate time moments to two adjacent supported discrete values.

According to the $M / M / 1$ queuing model, function $R_{i}=g\left(\mu_{i}, \lambda_{i}\right)$ is approximated as follows:

$$
R_{i}=\frac{1}{\mu_{i}-\lambda_{i}}=\frac{1}{\alpha_{i} f_{i}-\lambda_{i}}
$$

To guarantee $R_{i} \approx \hat{R}$, we approximate the proper $f_{i}$ to be:

$$
f_{i}=\frac{\lambda_{i}}{\alpha_{i}}+\frac{1}{\alpha_{i} \hat{R}}
$$

\footnotetext{
${ }^{1}$ An admission control mechanism could be applied to enforce this constraint.
} 
when $0<\lambda_{i} \leq \alpha_{i} f_{i_{-} \max }-\frac{1}{\hat{R}}$. This approximation, however, may introduce modeling inaccuracy. To overcome this inaccuracy, we combine feedback control with queuing-theoretic prediction for the dynamic voltage scaling (DVS). Nevertheless, in Section 6.5, experimental data shows that the queuing model estimate (Equation (6)) is very close to the real $f_{i}$ setting of the combined approach. This close approximation justifies the adoption of the queuing estimated $f_{i}$ in the problem formulation. The power management problem becomes:

minimize

$$
J=\sum_{i=1}^{N} x_{i}\left[c_{i}+\beta_{i} \times\left(\frac{\lambda_{i}}{\alpha_{i}}+\frac{1}{\alpha_{i} \hat{R}}\right)^{3}\right]
$$

\section{subject to:}

$$
\begin{cases}\sum_{i=1}^{N} x_{i} \lambda_{i}=\lambda_{\text {cluster }} & \\ x_{i}\left(1-x_{i}\right)=0, & i=1,2, \ldots, N \\ 0 \leq \lambda_{i} \leq \alpha_{i} f_{i \_m a x}-\frac{1}{\hat{R}}, & i=1,2, \ldots, N\end{cases}
$$

As shown above, the optimal solution is determined by two variables: individual server's on/off state $x_{i}$ and workload distribution $\lambda_{i}$. To achieve the optimal power consumption and to guarantee the average response time, the key therefore lies in the front-end, i.e., the power on/off and workload distribution strategies. We present these strategies in the next section.

\section{Algorithms}

When we design the power management strategies, one major focus is on their efficiencies. For a given workload $\lambda_{\text {cluster }}$, the front-end power management needs to decide 1) how many and which back-end servers should be turned on and 2) how much workload should be distributed to each server. Since $\lambda_{c l u s t e r}$ changes from time to time, these decisions have to be reevaluated and modified regularly. Thus, the decision process has to be very efficient.

The mechanism we propose is built on a sophisticated but low-cost offline analysis. It provides an efficient threshold-based online strategy. Assuming $\hat{\lambda}_{\text {cluster }}$ is the maximum workload that can be handled by the cluster without violating the average response time requirement. The offline analysis generates thresholds $\Lambda_{1}, \Lambda_{2}, \cdots, \Lambda_{N}$ and divides $\left(0, \hat{\lambda}_{\text {cluster }}\right]$ into ranges $\left(0, \Lambda_{1}\right],\left(\Lambda_{1}, \Lambda_{2}\right], \cdots,\left(\Lambda_{k}, \Lambda_{k+1}\right], \cdots,\left(\Lambda_{N-1}, \Lambda_{N}\right]$ (where $\left.\Lambda_{N}=\hat{\lambda}_{\text {cluster }}\right)$. For each range, the power on/off and workload distribution decisions are made offline. Dynamically the system just measures and predicts the workload $\lambda_{\text {cluster }}$, decides the range $\lambda_{\text {cluster }}$ falls into, and follows the corresponding power management decisions. Next, we present the details of our algorithm.

\subsection{Optimization Heuristic Framework}

In Section 4, the power management is formed as an optimization problem (Equations (7) and (8)). Instead of solving it for all possible workload $\lambda_{\text {cluster }}$ in the range $\left(0, \hat{\lambda}_{\text {cluster }}\right]$, we propose a heuristic to simplify the 
problem. It is constructed with the following framework:

- The heuristic first orders the heterogeneous back-end servers. It gives a sequence, called ordered server list, for activating machines. To shut down machines, the reverse order is followed.

- Second, the optimal thresholds $\Lambda_{k}, k \in\{1,2,3, \cdots N\}$ for turning on and off servers are identified: if $\lambda_{\text {cluster }}$ is in the range $\left(\Lambda_{k-1}, \Lambda_{k}\right]$, it is optimal to turn on the first $k$ servers of the ordered server list. This also means if $\lambda_{\text {cluster }}$ changes between adjacent ranges, such as from $\left(\Lambda_{k-1}, \Lambda_{k}\right]$ to $\left(\Lambda_{k}, \Lambda_{k+1}\right]$, the heuristic requires on/off state change for just one machine. Considering the high overhead of turning on/off servers (e.g., tens of seconds), this approach is superior in that it minimizes the server on/off state changes.

- Third, the optimal workload distribution problem is solved for $N$ scenarios where $\lambda_{\text {cluster }} \in\left(\Lambda_{k-1}, \Lambda_{k}\right], k=$ $1,2, \cdots, N$. When $\lambda_{\text {cluster }} \in\left(\Lambda_{k-1}, \Lambda_{k}\right]$, it is optimal to turn on the first $k$ servers of the ordered server list, i.e., $x_{i}=1, i=1,2, \cdots k$ and $x_{i}=0, i=k+1, k+2, \cdots, N$. With values of $x_{i}$ fixed, the optimization problem (Equations (7) and (8)) becomes:

minimize

$$
J_{k}=\sum_{i=1}^{k}\left[c_{i}+\beta_{i} \times\left(\frac{\lambda_{i}}{\alpha_{i}}+\frac{1}{\alpha_{i} \hat{R}}\right)^{3}\right]
$$

\section{subject to:}

$$
\left\{\begin{array}{l}
\sum_{i=1}^{k} \lambda_{i}=\lambda_{\text {cluster }} \\
0 \leq \lambda_{i} \leq \alpha_{i} f_{i_{-} \max }-\frac{1}{\hat{R}}, \quad i=1,2, \ldots, k
\end{array}\right.
$$

The analysis is simplified to solving the above optimization problem for $k=1,2, \cdots, N$.

Time complexity Analysis. If we consider solving the optimization problem (Equations (9) and (10)) as the basic operation, the time complexity of the proposed heuristic is $\Theta(N)$, while the time complexity to obtain the optimal power management solution (i.e., solving Equations (7) and (8) by an exhaustive search of all possible server on/off scenarios) for every integer point in the range $\left(0, \hat{\lambda}_{\text {cluster }}\right]$ is $\Theta\left(\left\lceil\hat{\lambda}_{\text {cluster }}\right\rceil 2^{N}\right)$.

In the next three subsections, we discuss the decisions on ordered server list, server activation thresholds and workload distribution respectively. For each decision, several strategies are investigated.

\subsection{Ordered Server List}

Our algorithm follows a specific order to turn on and off machines. To optimize the power consumption, this order must be based on the server's power efficiency, which is defined as the amount of power consumed per unit of workload (i.e., $\frac{P_{i}(\lambda)}{\lambda}$ ). Servers with better power efficiencies are listed first.

According to the power model and the dynamic voltage scaling mechanism adopted by back-end servers (Sections $3 \& 4$ ), the power consumption $P_{i}(\lambda)$ of a server includes a constant part $c_{i}$ and a variable part 
$\beta_{i} \times\left(\frac{\lambda}{\alpha_{i}}+\frac{1}{\alpha_{i} \hat{R}}\right)^{3}$ (see Equation (7)). Given any two servers $i$ and $j$, if $c_{i} \leq c_{j}$ and $\frac{\beta_{i}}{\alpha_{i}^{3}} \leq \frac{\beta_{j}}{\alpha_{j}^{3}}$, server $i$ has a better power efficiency than server $j$. However, if $c_{i}<c_{j}$ and $\frac{\beta_{i}}{\alpha_{i}^{3}}>\frac{\beta_{j}}{\alpha_{j}^{3}}$, the power efficiency order of the two is not fixed. When the server workload $\lambda$ is small, $P_{i}(\lambda)$ is less than $P_{j}(\lambda)$ and server $i$ has a better power efficiency; while as $\lambda$ increases, $P_{i}(\lambda)$ gets larger than $P_{j}(\lambda)$ and server $j$ 's power efficiency becomes better. In the proposed method, to trade for online algorithm's efficiency and minimum server on/off operations, the ordered server list is determined offline and is not subject to dynamic changes. Therefore, even if the servers' power efficiency order is not fixed, their activation order is nevertheless determined statically. Next we present our method and list several alternatives for generating the activation order.

- Typical Power based policy (TP). We assume the typical workload for a server is $\lambda_{i}^{\prime}$. In our heuristic, servers are ordered by their power consumption efficiency under the typical workload, i.e., $\frac{P_{i}\left(\lambda_{i}^{\prime}\right)}{\lambda_{i}^{\prime}}$. A server with smaller $\frac{P_{i}\left(\lambda_{i}^{\prime}\right)}{\lambda_{i}^{\prime}}$, i.e., smaller $\frac{c_{i}+\beta_{i} \times\left(\frac{\lambda_{i}^{\prime}}{\alpha_{i}}+\frac{1}{\alpha_{i} R}\right)^{3}}{\lambda_{i}^{\prime}}$, is listed earlier in the ordered server list. A power management mechanism usually turns on a server when needed or when it leads to a reduced power consumption (see Section 5.3). As a result, an active server usually works under a high workload. Thus we choose a workload that requires $80 \%$ capacity of a server as its typical workload $\lambda_{i}^{\prime}$. This way the ordered server list is created by comparing $\frac{P_{i}\left(\lambda_{i}^{\prime}\right)}{\lambda_{i}^{\prime}}$ and is solely based on the server's static parameters $\alpha_{i}, c_{i}$, and $\beta_{i}$.

- Activate All policy (AA). This activation policy always turns on all back-end servers. Therefore in this case the power on/off mechanism is not needed. Neither is the ordered server list.

- RANdom policy (RAN). This policy generates a random ordered server list for server activation.

- Static Power based policy (SP). This policy orders machines by their static power consumption. A server with a smaller static power consumption $c_{i}$ is listed earlier in the ordered server list.

- Pseudo Dynamic Power based policy (PDP). This policy orders machines by the dynamic power consumption parameter $\beta_{i}$. A server with a smaller $\beta_{i}$ is listed earlier in the ordered server list. According to the definition of power efficiency $\frac{P_{i}\left(\lambda_{i}\right)}{\lambda_{i}}$, its dynamic part is $\frac{\frac{\beta_{i}}{\alpha_{i}^{3}} \times\left(\lambda_{i}+\frac{1}{\tilde{R}}\right)^{3}}{\lambda_{i}}$. As we can see, the dynamic power efficiency is not solely determined by $\beta_{i}$. This policy is therefore called pseudo dynamic power based policy.

\subsection{Server Activation Thresholds}

In the previous section we introduced the ordered server list that specifies which servers to choose when we need to turn on or off machines. This section presents our threshold-based strategy to decide the optimal number of active servers.

The goal is two-fold. First, an adequate number of servers should be turned on to guarantee the response time requirement. Second, the number of active servers should be optimal with respect to the consumed power. 
To meet the response time requirement, the number of active servers should increase monotonically with the workload $\lambda_{\text {cluster. }}$ The heavier the workload, the greater the number of active servers required. It suggests that we turn on more servers only when the current capacity becomes inadequate to process the workload. Accordingly $N$ capacity thresholds $\Lambda_{c 1}, \Lambda_{c 2}, \cdots, \Lambda_{c N}$ are developed and each $\Lambda_{c k}$ corresponds to the maximum workload that can be processed by the first $k$ servers. According to Equation (5), when a server is operating at its maximum frequency $f_{i_{-} \max }$, it can process at most $\lambda_{i_{-} \max }$ amount of workload and meet the response time requirement:

$$
\lambda_{i \_\max }=\alpha_{i} f_{i \_\max }-\frac{1}{\hat{R}}
$$

Thus, we have:

$$
\Lambda_{c k}=\sum_{i=1}^{k} \lambda_{i \_\max }=\sum_{i=1}^{k} \alpha_{i} f_{i \_\max }-\frac{k}{\hat{R}}
$$

When the current workload exceeds this threshold $\Lambda_{c k}$, at least $k+1$ servers of the ordered server list have to be activated.

However, the above thresholds may not be optimal with respect to the power consumption. The power consumed by a server is composed of two parts: the static part $c_{i}$ and the dynamic part $\beta_{i} f_{i}^{3}$. When adding an active server, the cluster's static power consumption increases but its dynamic power consumption may actually decrease. The reason is that with more active servers to share the workload, the workload distributed to each server decreases; consequently, the CPU operating frequency $f_{i}$ required for each server may get smaller, which could lead to a reduced dynamic power consumption of the cluster.

To derive the optimal-power threshold, scenarios when activating $k+1$ servers is better than activating $k$ servers are identified. In such scenarios, $k$ servers are adequate to handle the workload. But if we activate $k+1$ servers, the system consumes less power. We assume that the optimal power consumption using the first $k$ servers to handle $\lambda_{\text {cluster }}$ workload, where $\lambda_{\text {cluster }} \in\left(0, \Lambda_{\text {ck }}\right]$, is $\hat{J}_{k}\left(\lambda_{\text {cluster }}\right)$ (see Section 5.4 for $\hat{J}_{k}\left(\lambda_{\text {cluster }}\right)$ 's derivation). It is a monotonically increasing function of $\lambda_{\text {cluster }}$. We analyze the following equation:

$$
\hat{J}_{k}\left(\lambda_{\text {cluster }}\right)=\hat{J}_{k+1}\left(\lambda_{\text {cluster }}\right)
$$

According to characteristics of functions $\hat{J}_{k}\left(\lambda_{\text {cluster }}\right)$ and $\hat{J}_{k+1}\left(\lambda_{\text {cluster }}\right)$ (see Section 5.4), there is at most one solution for Equation (13). If such a solution $\lambda_{\text {cluster }}^{\prime}$ is found, then activating $k+1$ servers is more power efficient than activating $k$ servers when $\lambda_{\text {cluster }}>\lambda_{\text {cluster }}^{\prime}$. Here is a sketch of the proof: 1$) \hat{J}_{k}\left(\lambda_{\text {cluster }}\right)$ is less than $\hat{J}_{k+1}\left(\lambda_{\text {cluster }}\right)$ for small $\left.\lambda_{\text {cluster }} ; 2\right)$ functions $\hat{J}_{k}\left(\lambda_{\text {cluster }}\right)$ and $\hat{J}_{k+1}\left(\lambda_{\text {cluster }}\right)$ increase monotonically with $\lambda_{\text {cluster }}$; and 3) if and only if $\lambda_{\text {cluster }}=\lambda_{\text {cluster }}^{\prime}$ activating $k$ or $k+1$ servers consumes the same amount of power. Therefore, once $\lambda_{\text {cluster }}$ exceeds $\lambda_{\text {cluster }}^{\prime}, \hat{J}_{k+1}\left(\lambda_{\text {cluster }}\right)$ becomes less than $\hat{J}_{k}\left(\lambda_{\text {cluster }}\right)$, i.e., it becomes 
more power efficient to activate $k+1$ servers.

Therefore, if there is a solution $\lambda_{\text {cluster }}^{\prime} \in\left(0, \Lambda_{c k}\right]$ for Equation (13), we find the optimal-power threshold $\Lambda_{p k}=\lambda_{\text {cluster }}^{\prime}$ where activating $k+1$ servers is more power efficient than activating $k$ servers when $\lambda_{\text {cluster }}$ exceeds this threshold; otherwise, we assign $\Lambda_{p k}=-1$. After analyzing Equation (13) for $k=1,2, \cdots, N-1$, we obtain another series of thresholds: optimal-power thresholds $\Lambda_{p 1}, \Lambda_{p 2}, \ldots, \Lambda_{p(N-1)}$.

By combining capacity and optimal-power thresholds, we get the server activation thresholds $\Lambda_{k}, k=$ $1,2, \cdots, N$ :

$$
\Lambda_{k}=\left\{\begin{array}{lr}
\Lambda_{c k} & \text { for } \Lambda_{p k}=-1 \text { or } k=N \\
\Lambda_{p k} & \text { for } \Lambda_{p k} \neq-1
\end{array}\right.
$$

We use the symbol $C P$ to denote the above Capacity-Power-based strategy. For comparison, a baseline $C A$ pacity-only strategy, denoted as $C A$, is also investigated, for which $\Lambda_{k}=\Lambda_{c k}$. In the Activate All policy (AA), no server activation thresholds are needed.

\subsection{Workload Distribution}

Last two sections solved the problem of deciding how many and which back-end servers should be activated for a given workload. This section proposes a strategy to optimally distribute the workload among active servers.

According to Section 5.1, if the first $k$ servers of the ordered server list are activated, the optimization problem becomes:

minimize

$$
J_{k}=\sum_{i=1}^{k}\left[c_{i}+\beta_{i} \times\left(\frac{\lambda_{i}}{\alpha_{i}}+\frac{1}{\alpha_{i} \hat{R}}\right)^{3}\right]
$$

\section{subject to:}

$$
\left\{\begin{array}{l}
\sum_{i=1}^{k} \lambda_{i}=\lambda_{\text {cluster }} \\
0 \leq \lambda_{i} \leq \alpha_{i} f_{\text {i_max }}-\frac{1}{\hat{R}}, \quad i=1,2, \ldots, k
\end{array}\right.
$$

The analysis is to find optimal solutions for all $J_{k}, k=1,2, \cdots, N$.

To solve the optimization for $J_{k}$, we first assume that all $k$ back-end servers are running below their maximum capacities, i.e, $0 \leq \lambda_{i}<\alpha_{i} f_{i_{-} \max }-\frac{1}{\hat{R}}, i=1,2, \ldots, k$. Since the second constraint of the problem is satisfied, the optimization becomes:

minimize

$$
J_{k}=\sum_{i=1}^{k}\left[c_{i}+\beta_{i} \times\left(\frac{\lambda_{i}}{\alpha_{i}}+\frac{1}{\alpha_{i} \hat{R}}\right)^{3}\right]
$$

subject to:

$$
\sum_{i=1}^{k} \lambda_{i}=\lambda_{\text {cluster }}
$$


According to Lagrange's Theorem [8], the first-order necessary condition for $J_{k}$ 's optimal solution is:

$$
\begin{aligned}
\exists \delta, J_{k}\left(\lambda_{i}, \delta\right)=\sum_{i=1}^{k}[ & \left.c_{i}+\beta_{i} \times\left(\frac{\lambda_{i}}{\alpha_{i}}+\frac{1}{\alpha_{i} \hat{R}}\right)^{3}\right] \\
+ & \delta\left(\sum_{i=1}^{k} \lambda_{i}-\lambda_{\text {cluster }}\right)
\end{aligned}
$$

and its first-order derivatives satisfy

$$
\left\{\begin{array}{l}
\frac{\partial J_{k}\left(\lambda_{i}, \delta\right)}{\partial \lambda_{i}}=0, \quad i=1, \ldots k \\
\frac{\partial J_{k}\left(\lambda_{i}, \delta\right)}{\partial \delta}=0
\end{array}\right.
$$

Solving the above condition, we obtain the optimal workload distribution $\lambda_{i}, i=1, \ldots, k$ as:

$$
\lambda_{i}=\frac{\alpha_{i}\left(\lambda_{\text {cluster }}+\frac{k}{\hat{R}}\right)}{\sum_{j=1}^{k} \alpha_{j} \sqrt{\frac{\alpha_{j}}{\beta_{j}}}} \sqrt{\frac{\alpha_{i}}{\beta_{i}}}-\frac{1}{\hat{R}}
$$

The corresponding power consumption is:

$$
\hat{J}_{k}=\sum_{i=1}^{k} c_{i}+\frac{\left(\lambda_{\text {cluster }}+\frac{k}{\hat{R}}\right)^{3}}{\left(\sum_{j=1}^{k} \alpha_{j} \sqrt{\frac{\alpha_{j}}{\beta_{j}}}\right)^{2}}
$$

The above solution is optimal when all $k$ back-end servers are running below their maximum capacities. That is, when $\lambda_{i}$ (Equation (20)) satisfies the constraint that $0 \leq \lambda_{i}<\alpha_{i} f_{i_{-} \max }-\frac{1}{\hat{R}}, i=1,2, \ldots, k$. Thus, the above condition holds true only for light cluster workloads. As $\lambda_{\text {cluster }}$ increases, servers start to be saturated one after another. That is, a server's shared workload $\lambda_{i}$ reaches its maximum level $\alpha_{i} f_{i_{-} \max }-\frac{1}{\hat{R}}$ where we have:

$$
\begin{aligned}
\lambda_{i} & =\frac{\alpha_{i}\left(\lambda_{\text {cluster }}+\frac{k}{\hat{R}}\right)}{\sum_{j=1}^{k} \alpha_{j} \sqrt{\frac{\alpha_{j}}{\beta_{j}}}} \sqrt{\frac{\alpha_{i}}{\beta_{i}}}-\frac{1}{\hat{R}} \\
& =\alpha_{i} f_{i_{-} \max }-\frac{1}{\hat{R}}
\end{aligned}
$$

Solving Equation (22) for system workload $\lambda_{\text {cluster }}$, we get:

$$
\lambda_{\text {cluster }}=f_{i_{-} \max } \sqrt{\frac{\beta_{i}}{\alpha_{i}}} \sum_{j=1}^{k} \alpha_{j} \sqrt{\frac{\alpha_{j}}{\beta_{j}}}-\frac{k}{\hat{R}}
$$

This result seems to indicate that among the $k$ active servers, the one with a smaller value of $f_{i_{-} \max } \sqrt{\frac{\beta_{i}}{\alpha_{i}}}$ reaches its full capacity earlier as $\lambda_{\text {cluster }}$ increases. We therefore order the $k$ servers by their $f_{i_{-} \max } \sqrt{\frac{\beta_{i}}{\alpha_{i}}}$ values and generate the saturated order list. When a server gets saturated, its shared workload should not be increased any more. Otherwise its response time $R_{i}$ will violate the requirement. As a result, after the first server's saturation, i.e., the saturation of the first server on the saturated order list, we have the server's shared 
workload as $\lambda_{1}=\alpha_{1} f_{1 \_m a x}-\frac{1}{\hat{R}}$ and the system workload as:

$$
\lambda_{\text {cluster }}=f_{1 \_\max } \sqrt{\frac{\beta_{1}}{\alpha_{1}}} \sum_{j=1}^{k} \alpha_{j} \sqrt{\frac{\alpha_{j}}{\beta_{j}}}-\frac{k}{\hat{R}}
$$

The workload distribution problem becomes:

minimize

$$
\begin{aligned}
J_{k} & =\sum_{i=2}^{k}\left[c_{i}+\beta_{i} \times\left(\frac{\lambda_{i}}{\alpha_{i}}+\frac{1}{\alpha_{i} \hat{R}}\right)^{3}\right] \\
& +\left(c_{1}+\beta_{1} f_{1 \_ \text {max }}^{3}\right)
\end{aligned}
$$

\section{subject to:}

$$
\sum_{i=2}^{k} \lambda_{i}=\lambda_{\text {cluster }}-\left(\alpha_{1} f_{1 \_ \text {max }}-\frac{1}{\hat{R}}\right)
$$

Here, servers are indexed following their saturated order list. Similar to Equations (16) and (17), we solve the above problem by applying Larange's Theorem and get the following optimal solution for $\lambda_{i}, i=2,3, \cdots, k$ :

$$
\lambda_{i}=\frac{\alpha_{i}\left(\lambda_{\text {cluster }}-\alpha_{1} f_{1 \_ \text {max }}+\frac{k}{\hat{R}}\right)}{\sum_{j=2}^{k} \alpha_{j} \sqrt{\frac{\alpha_{j}}{\beta_{j}}}} \sqrt{\frac{\alpha_{i}}{\beta_{i}}}-\frac{1}{\hat{R}}
$$

The corresponding power consumption is:

$$
\hat{J}_{k}=\sum_{i=1}^{k} c_{i}+\frac{\left(\lambda_{\text {cluster }}-\alpha_{1} f_{1 \_ \text {max }}+\frac{k}{\hat{R}}\right)^{3}}{\left(\sum_{j=2}^{k} \alpha_{j} \sqrt{\frac{\alpha_{j}}{\beta_{j}}}\right)^{2}}+\beta_{1} f_{1 \_\max }^{3}
$$

Again, we let $\lambda_{i}$ (Equation (27)) be equal to the maximum workload $\alpha_{i} f_{i_{-} m a x}-\frac{1}{\hat{R}}$ and solve for $\lambda_{c l u s t e r}$. We get:

$$
\lambda_{\text {cluster }}=f_{i \_\max } \sqrt{\frac{\beta_{i}}{\alpha_{i}}} \sum_{j=2}^{k} \alpha_{j} \sqrt{\frac{\alpha_{j}}{\beta_{j}}}+\alpha_{1} f_{1 \_\max }-\frac{k}{\hat{R}}
$$

This result verifies our hypothesis that servers saturate following the saturated order list — the smaller the value of $f_{i \_\max } \sqrt{\frac{\beta_{i}}{\alpha_{i}}}$, the earlier the server is saturated. The system workload that starts to saturate the first two servers is:

$$
\lambda_{\text {cluster }}=f_{2 \_ \text {max }} \sqrt{\frac{\beta_{2}}{\alpha_{2}}} \sum_{j=2}^{k} \alpha_{j} \sqrt{\frac{\alpha_{j}}{\beta_{j}}}+\alpha_{1} f_{1 \_ \text {max }}-\frac{k}{\hat{R}}
$$

We define $\lambda_{k}^{m}$ as:

$$
\lambda_{k}^{m}=f_{m_{-} \max } \sqrt{\frac{\beta_{m}}{\alpha_{m}}} \sum_{j=2}^{k} \alpha_{j} \sqrt{\frac{\alpha_{j}}{\beta_{j}}}+\sum_{i=1}^{m-1} \alpha_{i} f_{i_{-} \max }-\frac{k}{\hat{R}}
$$

In general, when $\lambda_{\text {cluster }} \in\left[\lambda_{k}^{m}, \lambda_{k}^{m+1}\right), m$ of the $\mathrm{k}$ active servers are saturated. That is, $\lambda_{i}=\alpha_{i} f_{i_{-} \max }-\frac{1}{\hat{R}}, i=$ 
$1,2, \cdots, m$. The optimization problem becomes:

minimize

$$
\begin{aligned}
J_{k} & =\sum_{i=m+1}^{k}\left[c_{i}+\beta_{i} \times\left(\frac{1}{\alpha_{i} \hat{R}}+\frac{\lambda_{i}}{\alpha_{i}}\right)^{3}\right] \\
& +\sum_{i=1}^{m}\left(c_{i}+\beta_{i} f_{i_{-} \max }^{3}\right)
\end{aligned}
$$

subject to:

$$
\sum_{i=m+1}^{k} \lambda_{i}=\lambda_{\text {cluster }}-\sum_{j=1}^{m}\left(\alpha_{j} f_{j \_ \text {max }}-\frac{1}{\hat{R}}\right)
$$

and the optimal solution is :

$$
\begin{aligned}
\lambda_{i}= & \frac{\alpha_{i}\left(\lambda_{\text {cluster }}-\sum_{j=1}^{m} \alpha_{j} f_{j \_ \text {max }}+\frac{k}{\hat{R}}\right)}{\sum_{j=m+1}^{k} \alpha_{j} \sqrt{\frac{\alpha_{j}}{\beta_{j}}}} \sqrt{\frac{\alpha_{i}}{\beta_{i}}}-\frac{1}{\hat{R}} \\
& \text { for } i=m+1, m+2, \cdots, k \\
\hat{J}_{k}= & \sum_{i=1}^{k} c_{i}+\frac{\left(\lambda_{\text {cluster }}-\sum_{j=1}^{m} \alpha_{j} f_{j \_ \text {max }}+\frac{k}{\hat{R}}\right)^{3}}{\left(\sum_{j=m+1}^{k} \alpha_{j} \sqrt{\frac{\alpha_{j}}{\beta_{j}}}\right)^{2}} \\
+ & \sum_{i=1}^{m} \beta_{i} f_{i_{-} \max }^{3}
\end{aligned}
$$

The above solution shows how to optimally distribute workload among active servers when they are in steady on states. However, since it takes some time (e.g., tens of seconds) to switch on a server and start software processes on it, there is a short server switch-on transient stage. During this transient interval, the to-be-active server cannot process any request yet, thus instead, the workload is distributed to active servers in proportion to their processing capacities. This temporary workload distribution method balances the load and avoids overloading the most power-efficient server during the transient stage. Once the transition is complete and the server is active and ready to process requests, the algorithm again begins to optimally distribute workload based on the aforementioned optimal solution.

Baseline Algorithms. We denote our algorithm proposed above as $O P$, the $O P$ timal workload distribution. For comparison, the following three baseline algorithms are investigated:

- RANdom (uniform) workload distribution (RAN). In this strategy, every incoming request is distributed 
to a randomly picked active server.

- CApacity based workload distribution (CA). This strategy distributes the workload among active servers in proportion to their processing capacities, i.e. $\alpha_{i} f_{i_{-} \max }$.

- One-by-One Saturation policy (OOS). This policy distributes requests following a default order. For each incoming request, we pick the first active server that is not saturated to process it.

\subsection{Algorithm Nomenclature}

The previous three subsections have respectively presented different strategies for deriving the ordered server list, server activation thresholds and workload distribution. By following the proposed framework (Section 5.1), we could generate many different algorithms by combining different strategies for the three modules, for instance, TP-CP-OP, AA-AA-CA and SP-CA-CA. The nomenclature of the algorithms includes three parts corresponding to the three design decisions. The first part denotes the adopted strategy for deciding the ordered server list: TP, AA, RAN, SP or PDP. The second part represents the choice for deriving server activation thresholds: CP, CA or AA. In the third portion of the name, OP, RAN, CA or OOS denotes the workload distribution strategy. However, not all combinations are feasible. For instance, CP can only be combined with $\mathrm{OP}$ and AA is combined with AA.

\section{Performance Evaluation}

In the previous section, we proposed various threshold-based strategies for the power management of heterogeneous soft real-time clusters. In this section, we experimentally compare their performance relative to each other, to an existing approach [17], and to the optimal solution.

A discrete simulator has been developed to simulate a range of heterogeneous clusters that are compliant to models presented in Section 3. The server on/off switch overheads are also simulated. There are two types of switch overheads: time overhead and power overhead. It takes some time, assumed to be 10 seconds, to turn on/off a server, during which interval no service can be provided by the server. To simulate the power overhead, we assume in the switch on/off interval, a server $S_{i}$ consumes power at the maximum level, i.e., $P_{i}=c_{i}+\beta_{i} f_{i_{-} \max }^{3}$

Cluster Configuration. The following clusters are simulated:

- Cluster1. First, we simulate a small cluster that consists of 4 back-end servers. They are all single processor machines: server 1 has an AMD Athlon $643000+1.8 \mathrm{GHz} \mathrm{CPU}$; server 2 has an AMD Athlon 64 X2 4800+ 2.4GHz CPU; server 3 has an Intel Pentium 4630 3.0GHz CPU and server 4 has an Intel Pentium D 950 3.4GHz CPU. To derive server parameters, experimental data from [17, 7, 10] are referred. Table 1 lists the estimated parameters. In addition, we assume that the processors only support discrete frequencies, i.e., a processor's frequency can only be set to one of ten discrete levels in the range $\left[f_{i_{-} \min }, f_{i_{-} \max }\right]$, where $f_{i_{-} \min }=25 \% f_{i_{-} \max }$. 


\begin{tabular}{|c|c|c|c|c|}
\hline Server & $f_{i-\max }$ & $c_{i}$ & $\beta_{i}$ & $\alpha_{i}$ \\
\hline 1 & 1.8 & 44 & 2.915 & 495.00 \\
\hline 2 & 2.4 & 53 & 4.485 & 548.75 \\
\hline 3 & 3.0 & 70 & 2.370 & 287.00 \\
\hline 4 & 3.4 & 68 & 3.206 & 309.12 \\
\hline
\end{tabular}

Table 1. Parameters of a 4-Server Cluster

- Cluster2. Second, we simulate a large cluster that has 128 back-end servers of 8 different types.

Workload Generation. A request is specified by a tuple $\left(A_{i}, E_{i}\right)$, where $A_{i}$ is its arrival time and $E_{i}$ is its execution time on a default server when it is operating at its maximum frequency.

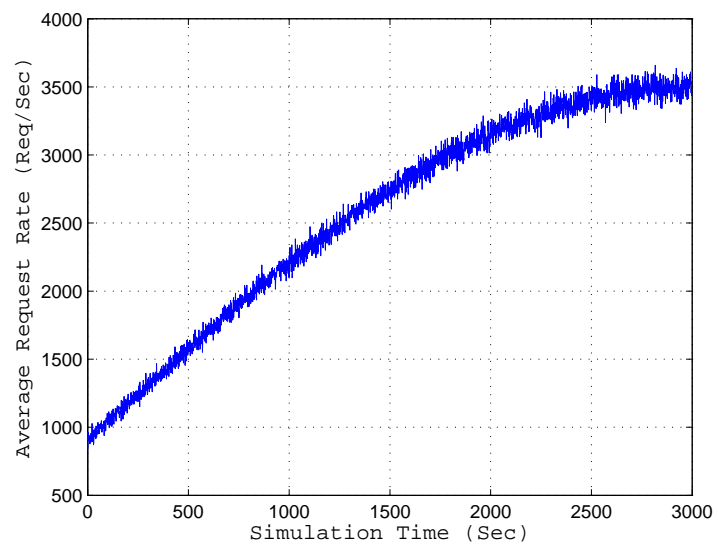

(a) Workload1

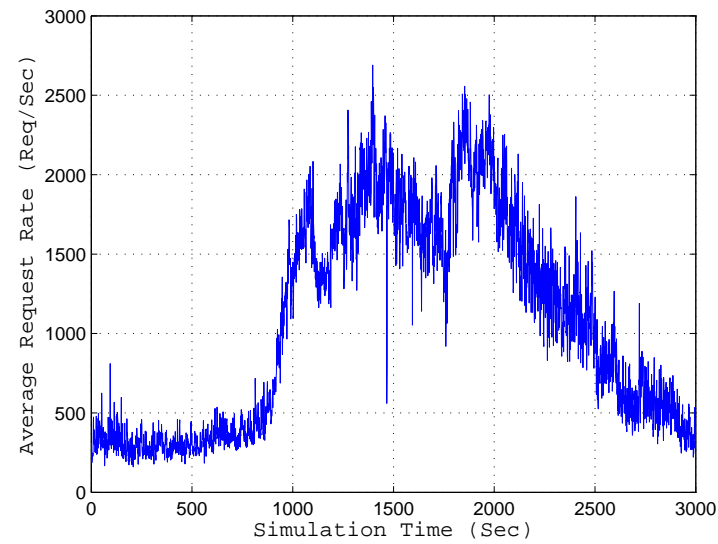

(b) Workload2

Figure 2. Average Request Rate

- To generate requests for Workload1, we assume that the inter-arrival time follows a series of exponential distributions with a time-varied mean of $\frac{1}{\lambda_{\text {cluster }}(t)}$. As shown in Figure 2a, we simulate a workload $\lambda_{\text {cluster }}(t)$ that gradually increases from requiring $20 \%$ to $90 \%$ of the cluster capacity. Request execution time $E_{i}$ is assumed to follow a gamma distribution with a specified mean of $\frac{1}{\mu_{1}^{\prime}}$, where $\mu_{1}^{\prime}=891 \mathrm{req} / \mathrm{sec}$ is the default server's maximum processing rate of this workload. The request execution time varies on different servers and is assumed to be reciprocally proportional to a server's capacity. Assuming small requests, their desired average response time $\hat{R}$ is set at 1 second.

- Workload2 is generated according to empirical distributions based on a server log file [19]. From the $\log$ file, we extract request arrival time and requested file size information. The log file records all requests that arrived in a day. To expedite the simulation, we replay these requests faster than real-time, i.e., we have proportionally reduced request interarrival times. The average request execution time is assumed to be $\frac{1}{\mu_{2}^{\prime}}$, where $\mu_{2}^{\prime}=541 \mathrm{req} / \mathrm{sec}$ is the default server's maximum processing rate of this workload. In addition, we assume request execution time $E_{i}$ grows linearly with requested file size. To simulate same application accesses, we choose requests with modest execution time variances, i.e., 
those $77.3 \%$ requests recorded in the log file whose execution times fall in the range $\left[\frac{0.1}{\mu_{2}^{\prime}}, \frac{10}{\mu_{2}^{\prime}}\right]$. Figure $2 b$ shows the generated request rate $\lambda_{\text {cluster }}(t)$.

By offline analysis of cluster server parameters, a threshold-based algorithm derives the ordered server list, server activation thresholds and workload distribution formulas. Once these three modules are deployed on the head node, the cluster is able to handle different levels of workload. Each simulation lasts 3000 seconds and periodically, i.e., every 30 seconds, the system measures the current workload and predicts the average request rate $\lambda_{\text {cluster }}(t)$ for the next period. We adopt a method proposed in [11] for the workload prediction. Based on the range the predicted $\lambda_{\text {cluster }}(t)$ falls into, the corresponding power management decisions on server on/off $\left(x_{i}\right)$ and workload distribution $\left(\lambda_{i}\right)$ are followed. According to $\lambda_{i}$, the back-end server DVS mechanism decides the server's frequency setting $f_{i}$. Since a CPU only supports discrete frequencies, we approximate the desired continuous frequency $f_{i}$ by switching the CPU frequency between two adjacent discrete values, e.g., to approximate $2.65 \mathrm{GHz}$ frequency, during the 30 -second sampling period, the CPU frequency is first set at $2.4 \mathrm{GHz}$ for 11.25 seconds and then at $2.8 \mathrm{GHz}$ for 18.75 seconds. To evaluate algorithm performance, we measure two metrics: average response time and power consumption. Curves are used to show the average response time, while for clarity, we use bar figures to illustrate the power consumption.

The first group of simulations (Sections 6.1, 6.2 and 6.3) simulate Cluster1 with the synthetic Workload1. We evaluate the effects of major design choices and the corresponding algorithms in Sections 6.1 and 6.2. Section 6.3 compares threshold-based algorithms with an existing approach [17] and with the optimal solution. In Sections 6.4 and 6.5, we simulate Cluster1 with the empirical Workload2 and experimentally evaluate the feedback control mechanism's impact on the back-end server DVS. Section 6.6 reports the simulation results on Cluster2.

\subsection{Effects of Ordered Server List}

We first evaluate an algorithm's performance with respect to different policies in deciding the ordered server list. Our heuristic: Typical Power based policy (TP) and baseline strategies: Activate All policy (AA), Static Power based policy (SP) and Pseudo Dynamic Power based policy (PDP) are compared. We evaluate the following algorithms: TP-CA-CA, AA-AA-CA, SP-CA-CA and PDP-CA-CA. Except for AA-AA-CA, which activates all servers, the other algorithms only differ in the ordered server list but have the same capacity based (CA) strategies for deciding server activation thresholds and workload distribution. Figures 3 and 4 show the simulation results.

Since algorithms adopt capacity based (CA) strategies for deciding server activation thresholds and workload distribution, we can see from Figure 3 they all achieve the response time goal and keep the average response time around 1 second. One interesting observation is that the Activate All policy (AA) does not decrease the response time. The reason is on a back-end server, the local DVS mechanism always sets the 
CPU frequency at the minimum levels that satisfy the time requirement. Therefore, as long as the desired frequency levels are equal or above the CPU's minimum frequency $f_{i \_m i n}$, even though AA policy turns on all back-end servers, it does not lead to reduced response times. The simulation results also demonstrate that our approach in approximating a continuous frequency by switching CPU between its two adjacent supported discrete frequencies works as expected.

In Figure 4, we use a table to list the average power consumption achieved by different algorithms over the 100 sampling periods and bars to show the sampled power consumptions in different sampling periods as cluster workload changes. Algorithm TP-CA-CA, built on our Typical Power based policy (TP), always consumes the least power. It performs especially well at a low/medium cluster request rate when a good power management mechanism is needed the most. As workload increases, all back-end servers have to be activated and the algorithms begin to have similar

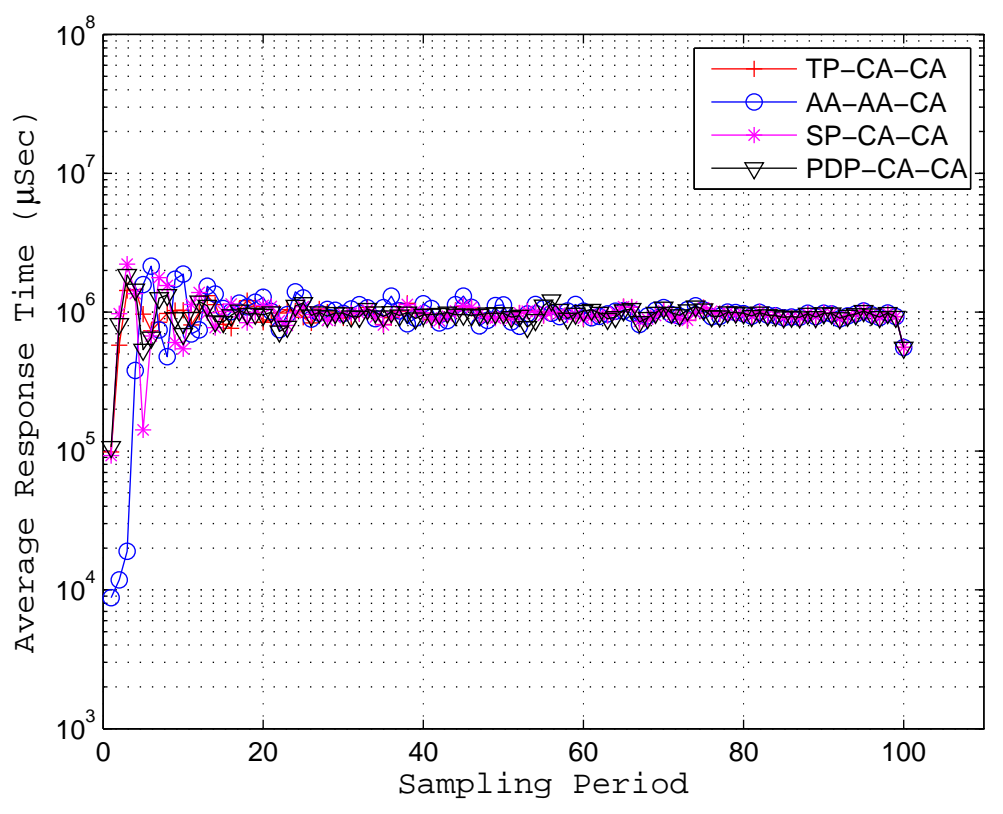

Figure 3. Effects of Ordered Server List: Time performance. From this experiment, we demonstrate that the server activation order has a big impact on the power efficiency. When adopting a bad order, such as that by the Pseudo Dynamic Power based policy (PDP), a high level of power is consumed. Occasionally, i.e., when $\lambda_{\text {cluster }}(t)=2457$ or $2747 \mathrm{req} / \mathrm{sec}$, the $P$ seudo Dynamic Power based policy (PDP-CA-CA) performs even worse than the Activate All policy (AA-AA-CA). It shows that under such scenarios activating more servers consumes less power.

\begin{tabular}{|c|c|}
\hline Alg & AvgPower (Watt) \\
\hline TP-CA-CA & 264 \\
\hline AA-AA-CA & 319 \\
\hline SP-CA-CA & 269 \\
\hline PDP-CA-CA & 306 \\
\hline
\end{tabular}

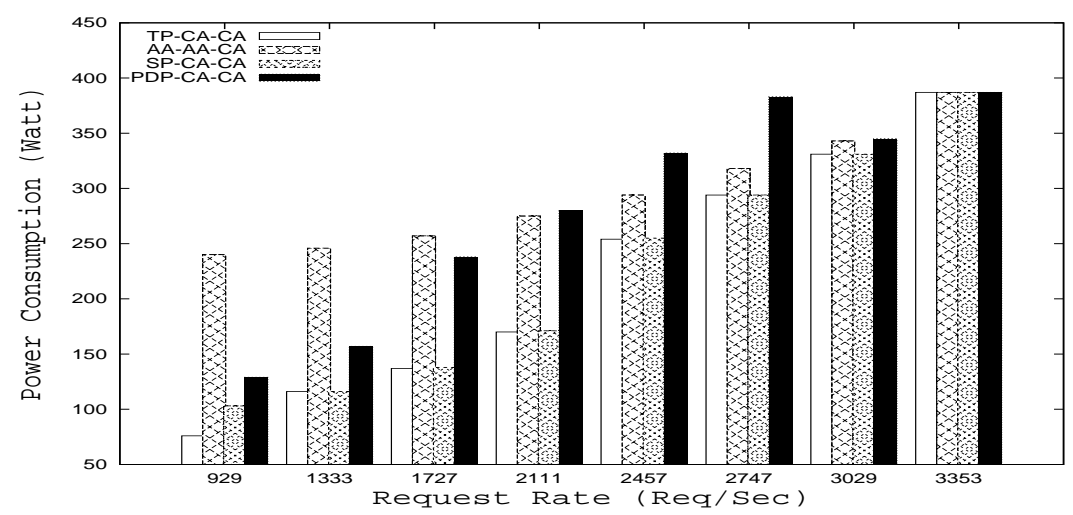

Figure 4. Effects of Ordered Server List: Power 


\subsection{Effects of Activation Thresholds and Workload Distribution}

In this subsection, to evaluate polices that decide server activation thresholds and workload distribution we simulate the following algorithms: RAN-CP-OP that is based on our heuristic and RAN-CA-OOS, RAN-CACA and RAN-CA-RAN baseline algorithms. For RAN-CP-OP, the last two modules are combined together since optimal-power thresholds depend on the optimal workload distribution. Therefore we evaluate the two polices together. For these algorithms, a common RANdomly generated ordered server list is used.

Figures 5 and 6 show the simulation results. From Figure 5, we can see that algorithm RAN-CA-RAN fails to provide response time guarantee: in multiple sampling periods, the average response time significantly exceeds the 1 second target. The reason is for a heterogeneous cluster, this RANdom (uniform) workload distribution does not prevent a server from being overloaded. Even though the CApacity-based server activation policy has ensured that the cluster capacity is adequate to handle the workload, the bad workload distribution still causes the QoS violation. Since all other algorithms consider a server's capacity for workload distribution, they meet the time requirement.

Figure 6 illustrates the power consumption results. Under all scenarios, the algorithm based on our heuristic, RAN-CP-OP, always consumes the least power. In addition, unlike other three algorithms, RAN-CP-OP's power consumption increases monotonically and smoothly with the workload. The main reasons behind these results are as follows. More Servers but Less Power. As discussed in Section 5.3, more servers do not always consume more power. Our Capacity-Powerbased strategy (CP) takes this factor into account. For example, when $\lambda_{\text {cluster }}(t)=929$

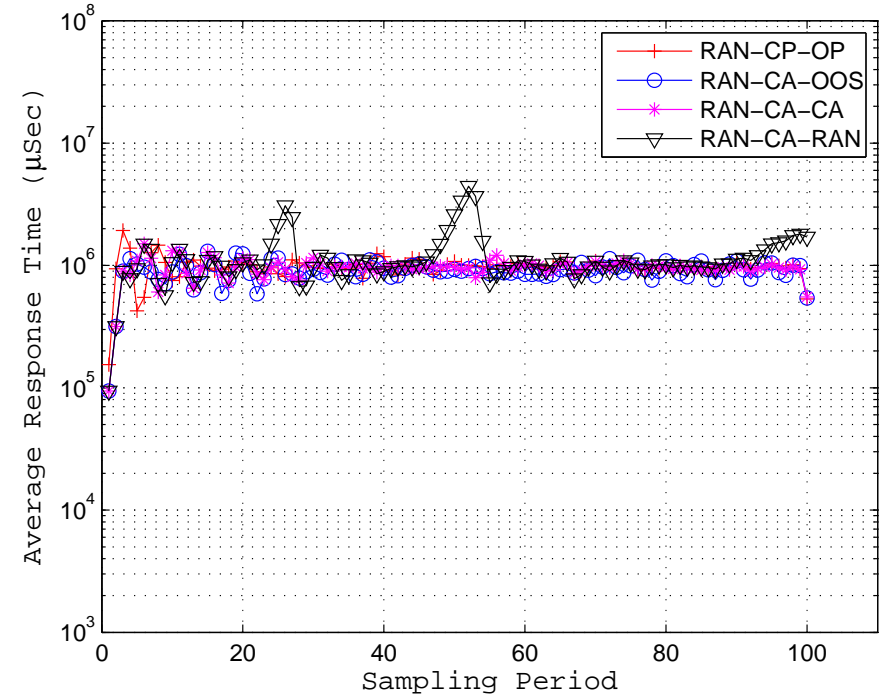

Figure 5. Effects of Activation Thresholds and Workload Distribution: Time

req/sec, the baseline $C A$ pacity-only based algorithms activate one server and when $\lambda_{\text {cluster }}(t)=2747 \mathrm{req} / \mathrm{sec}$, they activate three servers. In contrast, our algorithm RAN-CP-OP turns on two and four servers respectively under these two scenarios. It leads to much less power consumptions. When $\lambda_{\text {cluster }}(t)$ increases to $2800 \mathrm{req} / \mathrm{sec}$, RAN-CA-CA algorithm turns on the forth server. The result is that, with four servers its power consumption for a heavier workload (say $3029 \mathrm{req} / \mathrm{sec}$ ) is less than that of three servers for a lighter workload (say $2747 \mathrm{req} / \mathrm{sec}$ ). Optimal Workload Distribution. Our heuristic forms and solves the workload distribution as an optimization problem. The simulation results demonstrate that the resultant distribution is indeed optimal. In Figure 6, When $\lambda_{\text {cluster }}(t)$ is greater than $2800 \mathrm{req} / \mathrm{sec}$, four algorithms all activate the 
same number of servers. But our algorithm RAN-CP-OP still consumes the least power due to its optimal distribution of the workload. Unlike RAN-CP-OP, algorithm RAN-CA-OOS experiences a sudden change of the consumed power whenever a new server is activated. For this One-by-One Saturation strategy (OOS) on workload distribution, after adding an active server, its static power consumption increases but its dynamic power consumption does not decrease because it does not reduce the workload distributed to the other servers. Thus, their dynamic power consumptions do not decrease. As we observe, this strategy leads to the highest power consumptions.

\begin{tabular}{|c|c|}
\hline Alg & AvgPower (Watt) \\
\hline RAN-CP-OP & 278 \\
\hline RAN-CA-OOS & 364 \\
\hline RAN-CA-CA & 309 \\
\hline RAN-CA-RAN & 307 \\
\hline
\end{tabular}

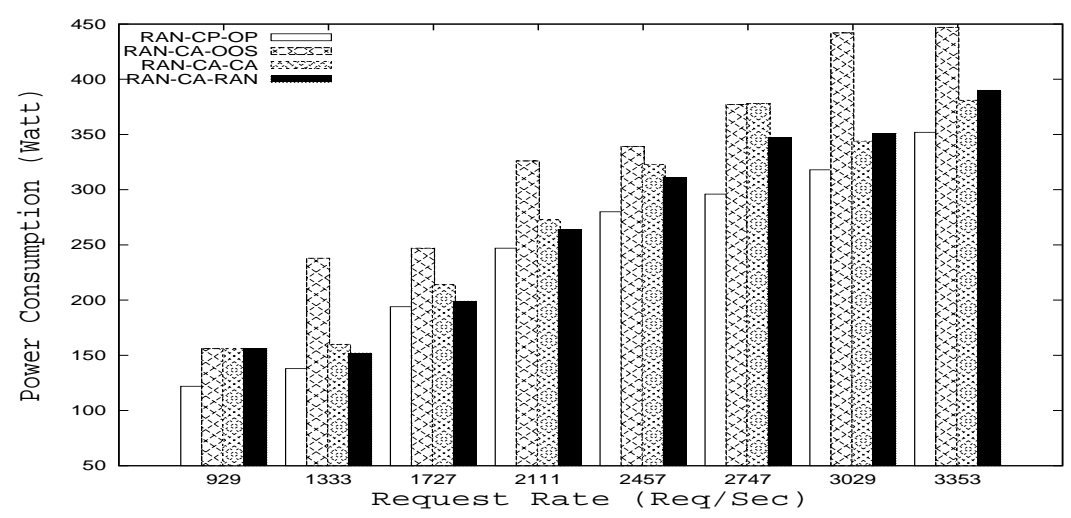

Figure 6. Effects of Activation Thresholds and Workload Distribution: Power

\subsection{Evaluation of Integrated Algorithms}

This subsection evaluates the following integrated algorithms: our heuristic TP-CP-OP and baseline algorithms: AA-AA-CA and SPCA-CA. When choosing baseline algorithms for comparison, we exclude the "deficient" algorithms, i.e., those based on PDP server activation strategy, RAN or OOS workload distribution policies. In addition, we compare these threshold-based algorithms with the optimal power management solution: OPTSOLN. To obtain the optimal solution, we solve the power management problem, i.e., Equations (7) and (8), for all integer points

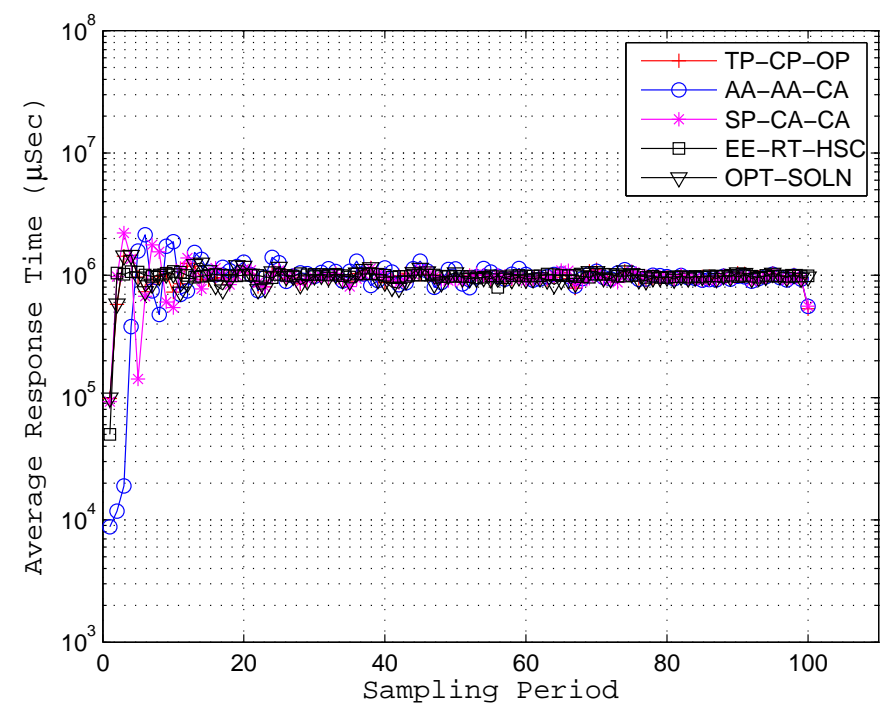

Figure 7. Integrated Algorithms: Time $\lambda_{\text {cluster }}$ in the range $\left(0, \hat{\lambda}_{\text {cluster }}\right]$. The optimal server on/off $\left(x_{i}\right)$ and workload distribution $\left(\lambda_{i}\right)$ is recorded for every possible $\lambda_{\text {cluster }}$. Dynamically, based on the predicted $\lambda_{\text {cluster }}(t)$, the corresponding optimal configuration is followed. We also implement an existing algorithm proposed by Rusu et. al. [17]. For that algorithm, since the authors simply assume servers can be easily ordered with respect to their power efficiencies, they 
only provide a very short discussion on the server activation order. Therefore, to compare that algorithm with our TP-CP-OP algorithm, we focus on the other two algorithmic decisions on: server activation thresholds and workload distribution, while adopting the same TP ordered server list for both algorithms. We denote that algorithm as EE-RT-HSC, which is the acronym of the paper's title [17].

\begin{tabular}{|c|c|}
\hline Alg & AvgPower (Watt) \\
\hline TP-CP-OP & 249 \\
\hline AA-AA-CA & 319 \\
\hline SP-CA-CA & 269 \\
\hline EE-RT-HSC & 257 \\
\hline OPT-SOLN & 254 \\
\hline
\end{tabular}

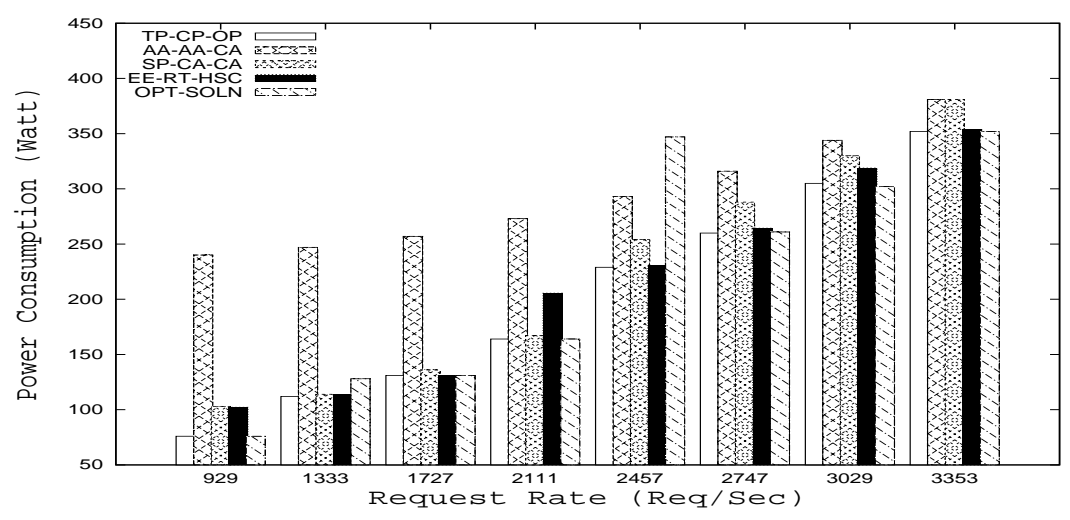

Figure 8. Integrated Algorithms: Power

Figures 7 and 8 respectively show the average response time and the power consumption. As expected, our algorithm TP-CP-OP performs better or as good as baseline algorithms under all scenarios. On average, TP-CP-OP consumes $28.1 \%$ and $8 \%$ less power than AA-AA-CA and SP-CA-CA respectively. Surprisingly, compared to the results of OPT-SOLN, our heuristic TP-CP-OP leads to an average of $2 \%$ less power consumption. For the simulated workload, OPT-SOLN algorithm switches on/off back-end servers for a total of 11 times, while our algorithm TP-CP-OP only turns on 3 additional servers at their individual appropriate moments following the ordered server list. During some sampling periods, OPT-SOLN algorithm may cause two server on/off switches, for instance, turning off a low-capacity server while turning on a high-capacity server at the same sampling period. In Figure 8 we observe when $\lambda_{\text {cluster }}(t)=2457 \mathrm{req} / \mathrm{sec}$, OPT-SOLN algorithm leads to a quite high power consumption, which is caused by two server switches in that sampling period. Following the threshold-based approach, our algorithm minimizes the server on/off overhead, resulting in a smaller power consumption. In comparison with EE-RT-HSC algorithm, on average, our TP-CP-OP algorithm consumes $3.2 \%$ less power. By analyzing the experimental data, we notice that these two algorithms switch on/off back-end servers for the same number times. However, two methods adopted by EE-RT-HSC lower the algorithm's power efficiency. First, to avoid overloading the cluster, the algorithm activates new servers in advance based on the possible maximum load increase during a monitoring period (which is set at 5 seconds). This strategy leads to servers being turned on too early, resulting in more power consumptions. Second, in order to void frequent server switches, EE-RT-HSC algorithm adds several transition states so that servers are not turned on/off immediately to improve power efficiency. This method, however, does not necessarily save power and in many cases, on the contrary, it leads to more power consumptions.

When implementing EE-RT-HSC, we notice that it is difficult to apply the algorithm to save power for web 
clusters that have fluctuating workloads. Web traffic is known to be self-similar [22] and thus has significant variability over a wide range of time scales. This huge variance makes it hard for the algorithm to estimate the possible maximum load increase per monitoring period. For instance, if we apply it to handle the empirical workload (i.e., Workload2 that we have generated based on a web log file), due to the large value of the max_load_increase, EE-RT-HSC algorithm will almost turn on all servers at the beginning of the simulation. As a result, it will consume a quite high level of power. Because of this deficiency, we choose not to simulate EE-RT-HSC in the next two subsections where the cluster executes the empirical Workload2.

\subsection{Empirical Workload Simulation}

To evaluate algorithms in a more realistic setting, for the second group of simulations (Sections 6.4 and 6.5), we simulate the cluster with the empirical Workload2, which is generated based on a web log file [19]. Figures 9 and 10 show the simulation results for the same integrated algorithms that have been analyzed in the previous subsection. From Figure 9, we can see, although the workload does not match the assumed M/M/1 queuing model, the algorithms can still keep response times around the 1 second target due to the effective feedback control of DVS. The only exception is for the

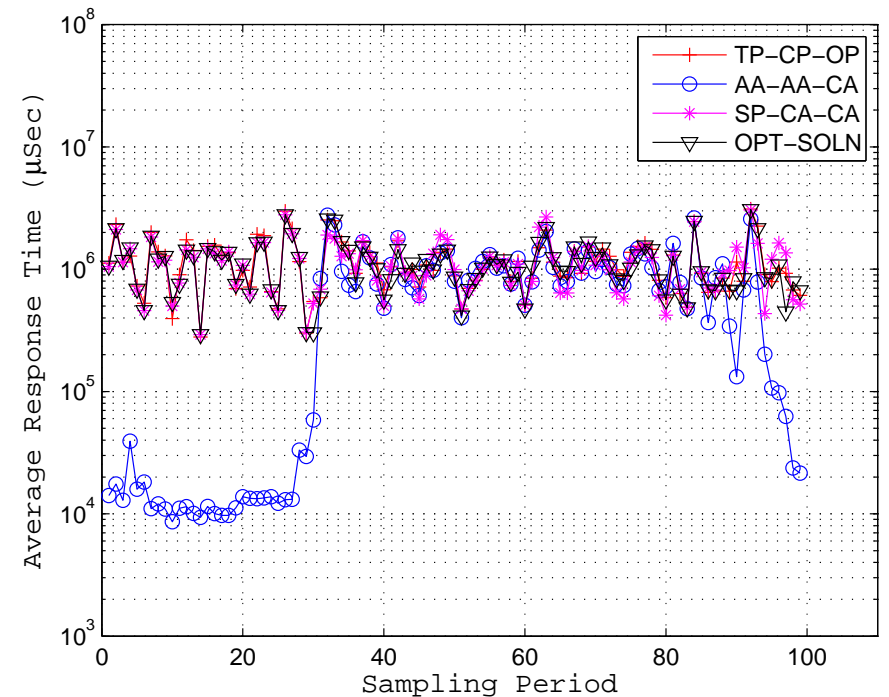

Figure 9. Simulation Result of Web Log Based Workload: Time

AA-AA-CA algorithm, which produces very short response times at the beginning and the end of the simulation. During those periods, the workload is very low, where $\lambda_{\text {cluster }}(t) \approx 500 \mathrm{req} / \mathrm{sec}$. However, AA-AA-CA algorithm always turns on all servers. With such low request rates, even when all processors are set at their minimum frequencies $f_{i_{-} m i n}$, the total cluster capacity is still bigger than needed to satisfy the 1 second response time requirement. That explains why we observe lower response times at the beginning and the end of the simulation.

Figure 10 again demonstrates that our algorithm TP-CP-OP achieves the best power efficiency. It outperforms baseline algorithms to a large extent. Furthermore, on average, TP-CP-OP consumes $2.9 \%$ less power than OPT-SOLN because the latter leads to a total of 28 server on/off switches, while our heuristic TPCP-OP only causes 10 switches. There are 9 sampling periods when OPT-SOLN algorithm causes 2 server switches and 10 sampling periods when it has 1 switch. During the two periods when $\lambda_{\text {cluster }}(t)=1360$ and $1397 \mathrm{req} / \mathrm{sec}$, our algorithm TP-CP-OP does not cause any server switch, while OPT-SOLN leads to 1 and 


\begin{tabular}{|c|c|}
\hline Alg & AvgPower (Watt) \\
\hline TP-CP-OP & 173 \\
\hline AA-AA-CA & 283 \\
\hline SP-CA-CA & 185 \\
\hline OPT-SOLN & 178 \\
\hline
\end{tabular}

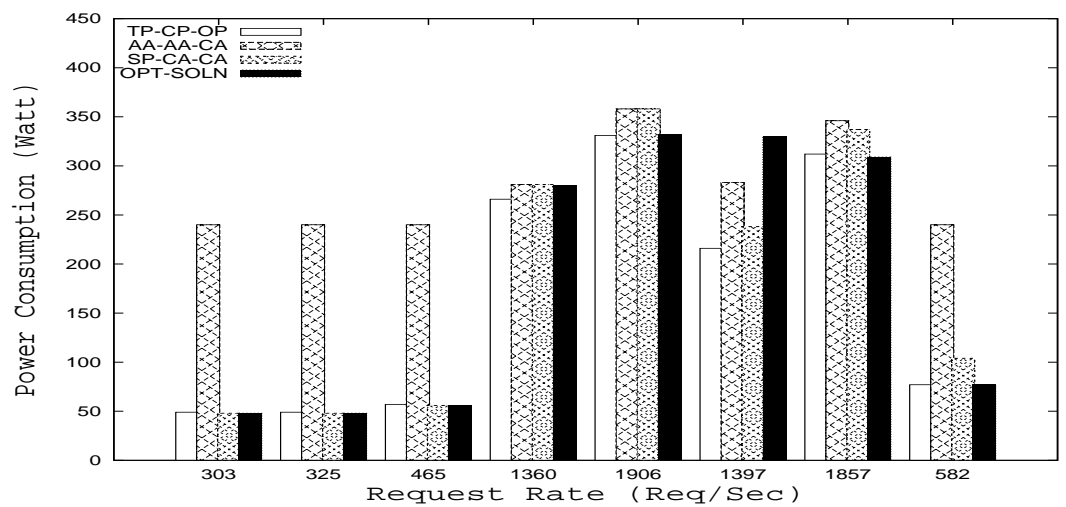

Figure 10. Simulation Result of Web Log Based Workload: Power

2 switches respectively. That is why during those periods OPT-SOLN algorithm consumes more power than TP-CP-OP.

\subsection{Effects of Feedback Control}

As described in Section 4, to overcome the inaccuracy of the $M / M / 1$ queuing model, we apply an approach that combines feedback control with queuing-theoretic prediction for back-end server DVS. This section evaluates the impact of the feedback control. We compare the combined mechanism with a queuing-theoretic prediction only mechanism where no feedback control of DVS is applied.

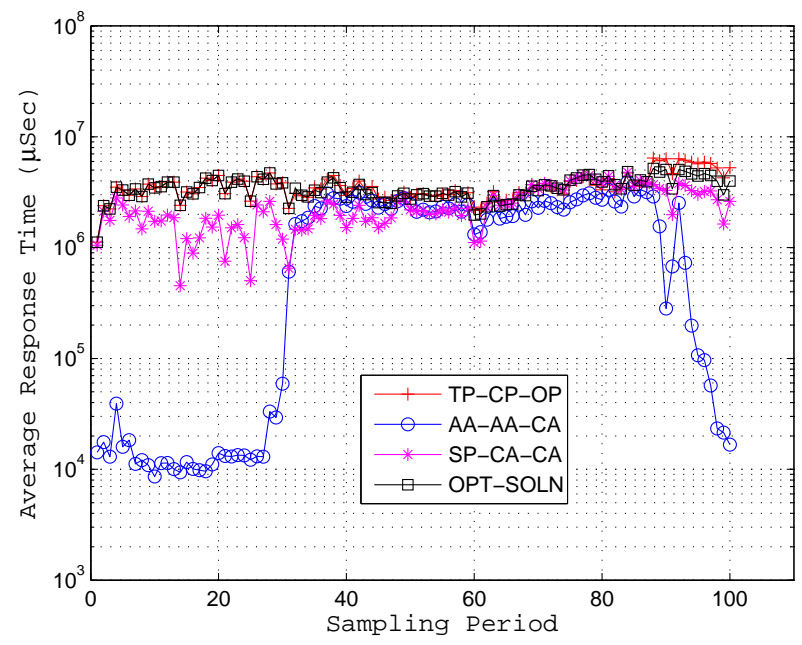

(a) Time

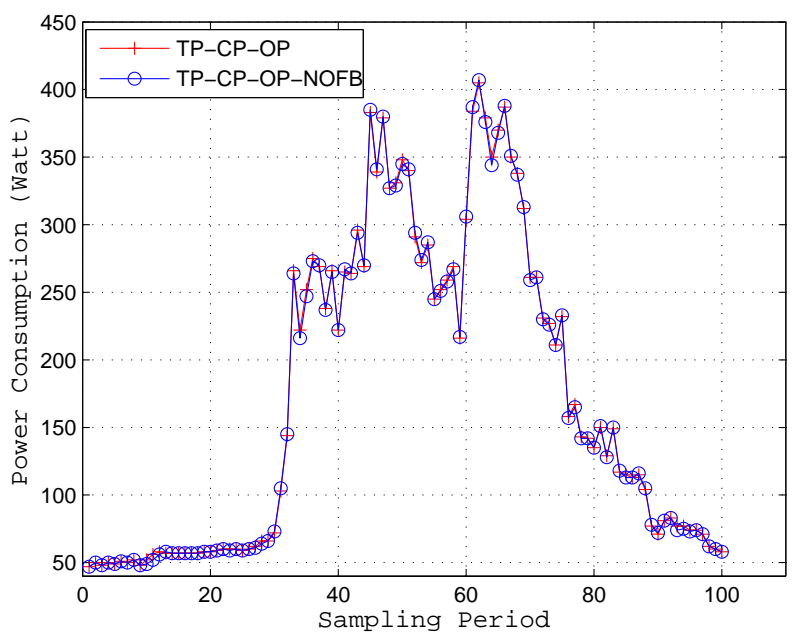

(b) Power

Figure 11. Effects of Feedback Control

Figure 11a shows the resultant average response times when the feedback control is not applied. As we can see, due to the modeling inaccuracy, the response times are no longer around the 1 second target. In contrast, when the feedback control is combined with the queuing-theoretic prediction, the average response times, as shown in Figure 9, are kept close to the target. These results demonstrate that the feedback control mechanism is effective in regulating the response time. On the other hand, when comparing power consumptions of DVS mechanisms with and without feedback control, the differences are negligible. For illustration, the curves in Figure $11 \mathrm{~b}$ show the power consumption of TP-CP-OP algorithm with and without DVS feedback control. On 
average, the difference in power consumption is only 0.22 Watt and server frequencies differ by $0.004 \mathrm{GHz}$. As we can see, the response time is very sensitive to frequency changes. A small frequency change can lead to a large variation of response time. Consequently, to effectively regulate the response time, the feedback control mechanism only needs to slightly modify the queuing estimated frequency $f_{i}$ and thus leads to a very small difference in power consumption. These experimental results show that the queuing model based estimate of $f_{i}$ is very close to the real frequency setting, which justifies the adoption of queuing estimated $f_{i}$ in the optimization problem formulation (see Section 4).

\subsection{Performance on a Large Cluster}

\begin{tabular}{|c|c|c|c|c|}
\hline Server & $f_{\text {i_max }}$ & $c_{i}$ & $\beta_{i}$ & $\alpha_{i}$ \\
\hline Type1 & 1.8 & 65 & 7.5 & 222.22 \\
\hline Type2 & 1.8 & 75 & 5 & 250.00 \\
\hline Type3 & 2.4 & 60 & 60 & 229.17 \\
\hline Type4 & 2.4 & 75 & 5.2 & 250.00 \\
\hline Type5 & 3.0 & 90 & 4.5 & 250.00 \\
\hline Type6 & 3.0 & 105 & 6.5 & 266.67 \\
\hline Type7 & 3.2 & 90 & 4.0 & 237.50 \\
\hline Type8 & 3.2 & 105 & 4.4 & 253.13 \\
\hline
\end{tabular}

Table 2. Parameters of a 128-Server Cluster

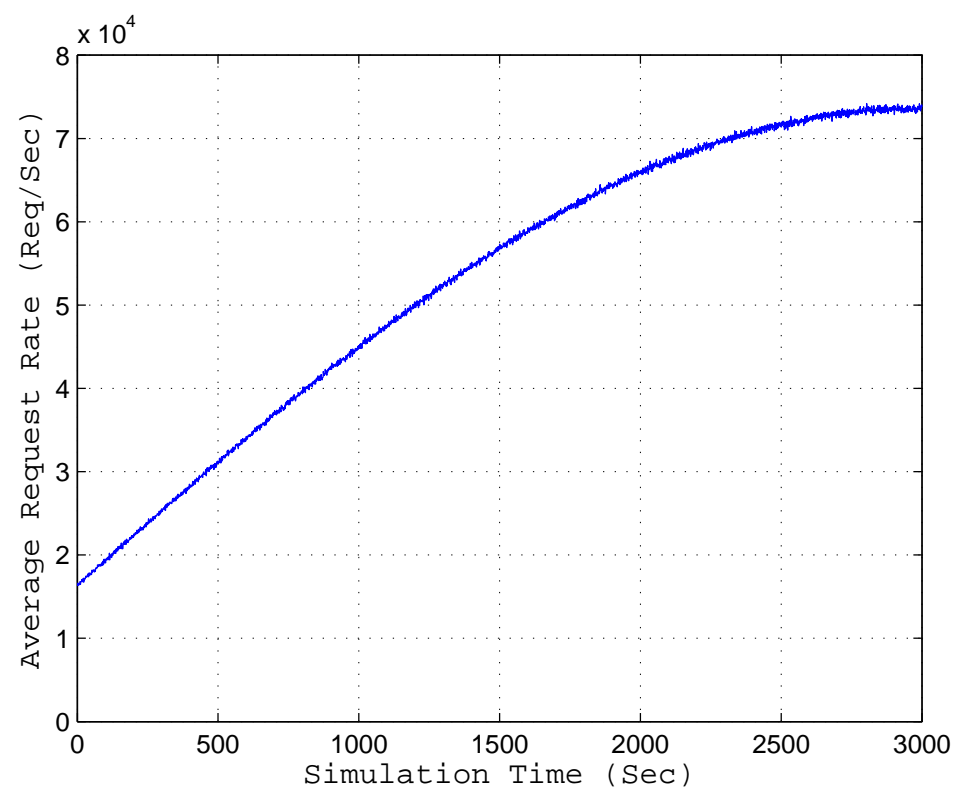

Figure 12. Large Cluster Simulation: Workload

In practice, a cluster typically runs with hundreds of back-end nodes. Therefore, in this subsection, we evaluate the algorithm's performance on a large cluster with 8 types of 128 nodes (see Table 2 for their parameters). 
Similar to Section 6.3, we compare three baseline algorithms: AA-AA-CA, SP-CA-CA and EE-RT-HSC with our heuristic: TP-CP-OP. Because OPT-SOLN algorithm has an exponential computation complexity, it cannot be implemented to handle large clusters. For that reason, OPT-SOLN is not included in this subsection. Figures 13 and 14 show the simulation results. As we can see, before the system workload reaches 56527 req/sec, AA-AA-CA consumes much more power than the other algorithms which include power on/off mechanisms. But as workload increases, AA-AA-CA outperforms SP-CA-CA algorithm. This result again proves that more servers do not always consume more power. Our algorithm TP-CP-OP considers both static and dynamic power efficiencies. Its mechanisms on power on/off and workload distribution strive to achieve an optimal power consumption. As a result, it performs the best. The algorithm EE-RT-HSC achieves approximately the same power consumption as our algorithm. The two algorithms have less than $0.35 \%$ difference in their power consumptions. However, compared to our TP-CP-OP algorithm, EE-RT-HSC takes a much longer time to finish its offline power management computation for such a large cluster. Besides, as discussed in Section 6.3, it is difficult to properly configure EE-RT-HSC to handle bursty empirical workloads. From Figure 13, we notice that at the beginning of the simulation, SP-AA-AA leads to higher average response times than the target. It is because as the workload quickly increases (see Figure 12 for the simulated synthetic workload), we need to turn on several servers. Due to the server activation overhead, there is a delay before a server can start processing requests. Because SP-CA-CA always activates as few back-end servers as possible, under this algorithm, active servers are running close to their maximum capacities before turning on more servers. As a result, during the transit period of turning on servers, increased workload saturates running servers. This overload effect is particularly obvious at the beginning of the simulation when only a small number of activated servers are sharing the excessive workload. On the other hand, TP-CP-OP and EE-RT-HSC consider the cluster power efficiency and always turn on more servers in advance. Consequently, their performance are not so sensitive to the server activation overhead.

\section{Conclusion}

This paper presents a threshold-based method for efficient power management of heterogeneous soft real-time clusters. Following this approach, a power management algorithm makes three important design decisions on ordered server list, server activation thresholds and workload distribution. We systematically study this approach and the impact of these design decisions. A new algorithm denoted as TP-CP-OP is proposed. When deciding the server activation order, the algorithm considers both static and dynamic power efficiencies. Its server activation thresholds and workload distribution are explicitly designed to achieve optimal power consumption. By simulation, we demonstrate the algorithm's advantages in power consumption: it incurs low overhead and leads to optimal power consumption.

\section{References}

[1] L. A. Barroso, The price of performance, Queue, September 2005, pp. 48-53 


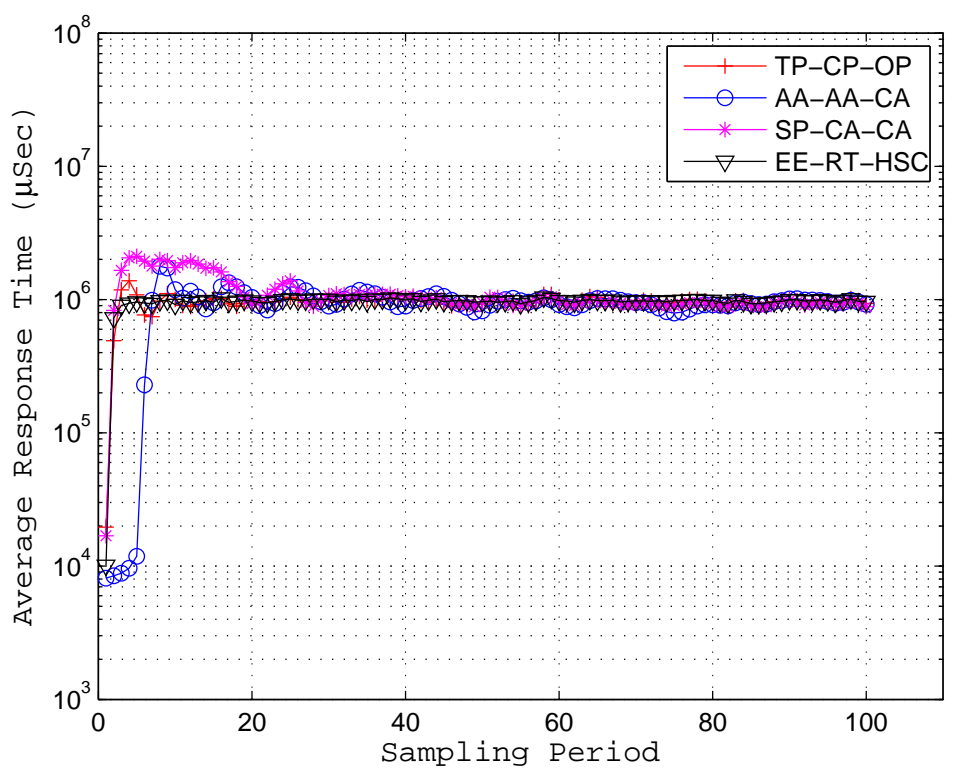

Figure 13. Simulation Result of Large Cluster: Time

\begin{tabular}{|c|c|}
\hline Alg & AvgPower (Watt) \\
\hline TP-CP-OP & 13434 \\
\hline AA-AA-CA & 15114 \\
\hline SP-CA-CA & 14182 \\
\hline EE-RT-HSC & 13481 \\
\hline
\end{tabular}

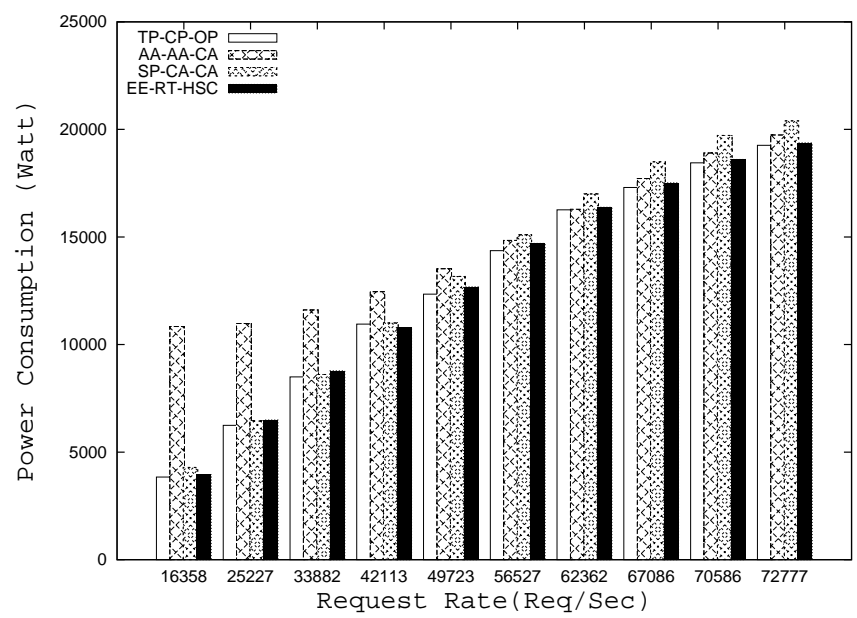

Figure 14. Simulation Result of Large Cluster: Power

[2] J. Chase, R. Doyle, Balance of power: energy management for server clusters, Proceedings of the 8th Workshop on Hot Topics in Operating Systems(HotOS-VIII), May 2001, pp. 163-165.

[3] V. Sharma, A. Thomas, T. Abdelzaher, K. Skadron, Z. Lu, Power-aware QoS management in web servers, In Proceedings of the 24th IEEE international Real-Time Systems Symposium, RTSS, IEEE Computer Society, Washington, DC, December 2003.

[4] R. Bianchini, R. Rajamony, Power and energy management for server systems, Computer 37(11)(2004)68-74.

[5] P. Bohrer, E. N. Elnozahy, T. Keller, M. Kistler, C. Lefurgy, C. McDowell, R. Rajamony, The case for power manage-management in web servers, Power Aware Computing, 2002, pp. 261-289.

[6] Y. Chen, A. Das, W. Qin, A.Sivasubramaniam, Q. Wang, N. Gautam, Managing server energy and operational costs in hosting centers, In SIGMETRICS'05: Proceedings of the 2005 ACM SIGMETRICS international conference on Measurement and modeling of computer systems, ACM Press, New York, NY, USA, 2005, pp. 303-314.

[7] M. Chin, Desktop cpu power survey, Silentpcreview.com, April 2006.

[8] E. Chong, S. H. Zak, An introduction to optimization, Wiley-Interscience, July 2001. 
[9] M. Elnozahy, M. Kistler, R. Rajamony, Energy-efficient server clusters, In Proceedings of the Second Workshop on Power Aware Computing Systems, February 2002.

[10] T. Hardware, Cpu performance charts, Tom's Hardware, 2006.

[11] J. P. Hayes, Self-optimization in computer systems via on-line control: Application to power management, In ICAC04: Proceedings of the First International Conference on Autonomic Computing (ICAC04), IEEE Computer Society, Washington, DC, USA, 2004, pp. 54-61.

[12] T. Heath, B. Diniz, E. V. Carrera, W. M. Jr., and R. Bianchini. Energy conservation in heterogeneous server clusters. In PPoPP 05: Proceedings of the tenth ACM SIGPLAN symposium on Principles and practice of parallel programming, ACM Press, New York, NY, USA, 2005, pp. 186-195.

[13] J. Heo, D. Henriksson, X. Liu, T. Abdelzaher, Integrating adaptive components: An emerging challenge in performanceadaptive systems and a server farm case-study, In 28th IEEE International Real-Time Systems Symposium, Tuscon, AZ, December 2007, pp. 227-238.

[14] L.Mastroleon, N.Bambos, C.Kozyrakis, D.Economou, Autonomic power management schemes for internet servers and data centers, In Proceedings of the IEEE Global Telecommunications Conference (GLOBECOM), 2005.

[15] E. Pinheiro, R. Bianchini, E. Carrera, T. Heath, Load balancing and unbalancing for power and performance in cluster-based systems, In Proceedings of the International Workshop on Compilers and Operating Systems for Low Power, May 2001.

[16] K. Rajamani, C. Lefurgy, On evaluating request-distribution schemes for saving energy in server clusters, In ISPASS 03: Proceedings of the 2003 IEEE International Symposium on Performance Analysis of Systems and Software, IEEE Computer Society, Washington, DC, USA, 2003, pp. 111-122.

[17] C. Rusu, A. Ferreira, C. Scordino, A. Watson, R. Melhem, D. Mosse, Energy-efficient real-time heterogeneous server clusters, In Proceedings of the Twelfth Real-Time and Embedded Technology and Applications Symposium (RTAS06), April 2006.

[18] L. Sha, X. Liu, Y. Lu, T. Abdelzaher, Queueing model based network server performance control, In IEEE RealTime Systems Symposium, Austin, TX, December 2002.

[19] ftp://ftp.ircache.net/Traces/DITL-2007-01-09/rtp.sanitized-access.20070109.gz

[20] Yefu Wang, Xiaorui Wang, Ming Chen, Xiaoyun Zhu, Power-efficient response time guarantees for virtualized enterprise servers, In Proceedings of the Real-Time Systems Symposium (RTSS08), Barcelona, Spain, December, 2008, pp. 303-312.

[21] Leping Wang, Ying Lu, Efficient power management of heterogeneous soft real-time clusters, In Proceedings of the Real-Time Systems Symposium (RTSS08), Barcelona, Spain, December, 2008, pp. 323-332.

[22] Mark E. Crovella, Azer Bestavros, Self-similarity in world wide web traffic evidence and possible causes, IEEE/ACM Transactions on Networking, 1996, vol. 5, pp. 835-846.

[23] Raphael Guerra, Julius Leite, Gerhard Fohler, Attaining soft real-time constraint and energy-efficiency in web servers, In Proceedings of the ACM symposium on Applied computing, 2008, pp. 2085-2089.

[24] Ming Xiong, Song Han, Kam-Yiu Lam, Deji Chen, Deferrable scheduling for maintaining real-time data freshness: algorithms, analysis, and results, IEEE Transactions on Computers, vol. 57, No. 7, 2008, pp. 952-964.

[25] Jian-Jia Chen, Andreas Schranzhofer, Lothar Thiele, Energy minimization for periodic real-time tasks on heterogeneous processing units, In Proceedings of International Parallel and Distributed Processing Symposium (IPDPS), Rome, Italy, May, 2009, pp. 1-12. 
[26] Luciano Bertini, Julius C. B. Leite, Daniel Mosse, Generalized tardiness quantile metric: distributed DVS for soft real-time web clusters, In Proceedings of the Euromicro Conference on Real-Time Systems (ECRTS09), July 2009, pp. 227-236. 